\title{
Paramètres phénotypiques et génétiques des caractères de crolssance et de reproduction des brebls croisées Berrichon du Cher $\times$ Romanov F1 à F4
}

\author{
G. RICORDEAU, J. RAZUNGLES, L. TCHAMITCHIAN, C. LEFEVRE \\ et J.C. BRUNEL
}

avec la collaboration technique de D. Lajous

I.N.R.A., Station d'Amélioration génétique des animaux

Centre de Recherches de Toulouse, B.P. 12, F 31320 Castanet-Tolosan

\section{Résumé}

Cette étude a pour but de comparer les performances de croissance et les aptitudes maternelles des brebis F1 à F4 issues du croisement entre les 2 races parentales Romanov et Berrichon du Cher. L'analyse des moindres carrés a été réalisée sur 1298 brebis nées de 1973 à 1977 et contrôlées jusqu'en 1979. Le système original de reproduction comporte une première lutte en mai à 14 mois (fertilité : 0,86 ), une $2^{\circ}$ lutte post-partum en octobre à 20 mois (fertilité : 0,82) et des luttes suivantes 1 fois par an en juillet-août (fert. 0,97 à 0,99). Les performances enregistrées aux mises bas 1 à 4 sont respectivement : 1,67 - 1,87 1,98 et 2,01 pour le nombre de nés totaux; $54,5-57,0-62,8$ et $65,6 \mathrm{~kg}$ pour le poids à la mise bas ; 1,53 - 1,66 - 1,95 et 2,01 kg de lait pour la quantité moyenne journalière de lait consommée par les agneaux allaités pendant le premier mois.

Les différences entre générations $F 1$ à $F 4$ sont significatives pour les poids à 70 et 90 jours (du fait des croissances inférieures en F1), mais non significatives pour le gain 30-70 jours. Elles sont non significatives pour le poids à la mise bas, la fertilité, le nombre d'agneaux nés et vivants par portée et la production laitière. Ce résultat confirme les observations antérieures obtenues sur brebis F1 et F2, nées de 1970 à 1972 et conduites en rythme traditionnel; il démontre l'absence de «régression» des performances de la F1 à la F4, dans nos conditions (race parentale Romanov, pas de sélection jusqu'à la F4).

Le type de naissance-allaitement des brebis a une influence significative sur le poids à la mise bas jusqu'à 4 ans et sur la prolificité à la mise bas 1 , donnant un avantage de 0,10 agneau aux brebis nées simples.

L'héritabilité des performances a été estimée séparément pour les brebis F2 et F3 par la méthode des demi-sceurs de pères. $L^{\prime} h^{2}$ du gain $30-70$ jours $(0,17$ et 0,43$)$ est supérieure à celle de la prolificité $(0,09$ et 0,24$)$ et de la production laitière $(0$ et 0,16$)$ à la mise bas 1 . La croissance moyenne de 30-70 jours des agnelles est le critère qui a la corrélation génétique la plus élevée avec la prolificité à la mise bas 1 .

\section{Introduction}

Parmi les différentes stratégies d'utilisation des races prolifiques (Tchamitchian et al., 1975, 1979), la création d'une souche synthétique permet d'atteindre une productivité élevée, tout en répondant aux préoccupations des éleveurs qui souhaitent 
une conduite simplifiée de leur troupeau (notamment grâce à l'autorenouvellement des femelles) avec des brebis ayant une prolificité voisine de 2 , possédant une bonne aptitude maternelle, et si possible, aptes au désaisonnement. Aussi, dès 1969, l'I.N.R.A. a mis en place un programme de création d'une souche croisée à partir des 2 races parentales Berrichon' du Cher et Romanov, dont les aptitudes bouchères et de reproduction sont complémentaires. Dans notre expérimentation, les premières F1 et F2 contemporaines naissent en 1970 (RICORDEAU et al., $1976 \mathrm{~d}$ ), mais cette analyse commence avec les femelles nées en 1973 qui ont été les premières à suivre le nouveau rythme de reproduction comportant 2 luttes consécutives de 1 à 2 ans, l'une à contre saison, l'autre post-partum en saison. Nous disposons des résultats obtenus sur les brebis des 4 premières générations reproduites sans sélection. Cette étude a pour but de comparer leurs performances de croissance, de reproduction et leur aptitude laitière, et d'estimer les paramètres génétiques de ces caractères, afin de faire le point sur la variabilité phénotypique et génétique dans une souche synthétique en voie de constitution. Ultérieurement, nous analyserons les aptitudes bouchères, les aspects génétiques liés à la coloration et nous présenterons la méthodologie de sélection applicable à la souche croisée à partir de la $4^{\circ}$ génération.

\section{Matériel et méthodes}

\section{Matériel animal}

Cette expérimentation a été conduite au Domaine de la Sapinière, près de Bourges ( ${ }^{\circ} 20^{\prime}$ de longitude Est, $47^{\circ} 05^{\prime}$ de latitude Nord). Elle concerne les brebis nées sur 5 campagnes (1973 à 1977) et appartenant aux 4 premières générations de brebis croisées (F1 à F4) issues des 2 races parentales Berrichon du Cher (BC) et Romanov (RO). La répartition des effectifs de brebis et de leurs pères est indiquée dans le tableau 1. Les brebis F1 sont issues des accouplements réciproques entre les reproducteurs des 2 races parentales. De même, les F2 sont issues des 2 types de reproducteurs F1. D'une façon générale, depuis le début de l'expérimentation, le programme des accouplements destinés à la production des animaux de chaque génération utilise des béliers issus de toutes les familles parentales de départ (11 familles Berrichonnes et 11 Romanov), chaque famille étant représentée par 1 ou plusieurs mâles suivant l'effectif des familles. Toutes les agnelles normalement développées sont conservées. Les béliers retenus pour la reproduction n'ont fait l'objet d'aucune sélection systématique et peuvent être considérés comme représentatifs des mâles contemporains de chaque génération, même si leur poids à 70 jours et la prolificité de leur mère à 3 et 4 ans sont légèrement supérieurs à la moyenne (tabl. 2).

\section{Rythme de reproduction}

Toutes les brebis sont conduites en agnelage accéléré jusqu'à 3 ans, selon le programme suivant : naissance en décembre-janvier ; $1^{\text {re }}$ lutte à 15 mois en avril-mai et agnelage en septembre-octobre ; $2^{\circ}$ lutte post-partum en octobre-novembre et agnelage en mars-avril ; $3^{*}$ lutte en juillet-août et agnelage en décembre-janvier à 3 ans ; les luttes suivantes se déroulent toujours en juillet-août, ce qui donne une $4^{e}$ mise bas à 4 ans et ainsi de suite. Les 2 premières luttes sont pratiquées sans contrôle de 


\section{TABleau 1}

Répartition des brebis par année de naissance et numéro de génération.

Distribution of ewes by birth-year and generation number.

\begin{tabular}{|c|c|c|c|c|c|}
\hline \multirow{2}{*}{$\mathrm{N}^{\circ}$ de génération } & \multicolumn{5}{|c|}{ Année de naissance des brebis } \\
\hline & 1973 & 1974 & 1975 & 1976 & 1977 \\
\hline F1 & $121(19)$ & $49(21)$ & 0 & $30(3)$ & 0 \\
\hline F2 & $86(15)$ & $116(19)$ & $71(25)$ & $129(19)$ & $128(20)$ \\
\hline F3 & $22(6)$ & $117(13)$ & $91(25)$ & $102(17)$ & $146(18)$ \\
\hline$\ldots$ & 0 & 0 & 0 & $20(6)$ & $70(10)$ \\
\hline $\begin{array}{l}\text { Mises bas prises en } \\
\text { compte } \ldots \ldots \ldots \ldots \ldots\end{array}$ & 1 à 4 & 1 à 4 & 1 à 4 & 1 à 4 & 1 à 3 \\
\hline
\end{tabular}

- Le nombre de pères des brebis par année de naissance-génération est indiqué entre parenthèses. - Année de naissance $\mathrm{i}=$ naissance du 15 décembre de l'année $(\mathrm{i}-1)$ au 15 février de l'année $\mathrm{i}$. Blocs de données

- $\mathrm{n}^{\circ} 1$ : $\mathrm{F} 1$ à $F 3$, naissances 1973 et $1974 ; 511$ brebis nées de 93 pères $X$ année.

- $\mathrm{n}^{\circ} 2$ : $\mathrm{F} 2$ et $\mathrm{F} 3$, naissances 1973 à $1977 ; 1008$ brebis nées de 177 pères $\times$ année.

- $\mathrm{n}^{\circ} 3$ : F2 à $\mathrm{F} 4$, naissances 1976 et $1977 ; 595$ brebis nées de 90 pères $\times$ année.

Total : F1 à F4, naissances 1973 à $1977 ; 1298$ brebis nées de 236 pères $\times$ année .

Nombre de pères différents des brebis $F 1$ à $F 4=146$ pères.

Brebis $F 1$ : 30 pères; $F 2: 46$ pères; $F 3: 53$ pères; $F 4: 17$ pères

\section{TABLEAU 2}

Poids à 70 jours et coloration des pères des brebis F3 et F4, et prolificité des grands-mères paternelles des brebis $F 3$ et F4.

Liveweight at 70 days and colour of sire of F3 and F4 ewes, and prolificacy of paternal-grand-mothers of F3 and F4 ewes.

\begin{tabular}{|c|c|c|c|c|c|}
\hline \multirow{2}{*}{ Brebis } & \multirow{2}{*}{$\begin{array}{l}\text { Nombre } \\
\text { de pères } \\
\text { différents }\end{array}$} & \multicolumn{2}{|c|}{ Pères des brebis $\mathrm{F} 3$ et $\mathrm{F} 4$} & \multicolumn{2}{|c|}{$\begin{array}{c}\text { Prolificité des GM } \\
\text { paternelles des brebis } \\
\text { F3 à F4 à }\end{array}$} \\
\hline & & $\begin{array}{l}\text { Poids à } 70 \mathrm{j} \\
(\mathrm{kg})\end{array}$ & $\begin{array}{l}\text { P. } 100 \text { de } \\
\text { colorés (*) }\end{array}$ & 3 ans & 4 ans \\
\hline F3 & 53 & $22 ; 8$ & 30 & 2,15 & 2,22 \\
\hline F4 & 17 & 20,7 & 33 & 2,20 & 2,17 \\
\hline
\end{tabular}

(*) Sur la totalité des agneaux nés, le pourcentage de colorés est de 31 en F2 et de 42 en F3. 
paternité, avec un groupe de béliers croisés, tous les agneaux étant destinés à la boucherie. La $3^{\text {e }}$ lutte et les suivantes sont réservées au renouvellement : du 15 juillet au 5 septembre environ, détection des œstrus par des béliers vasectomisés et saillies contrôlées; après le 5 septembre, rattrapage des brebis non fécondées en lutte libre, avec un groupe de béliers. Afin de limiter les sources de variation dans l'analyse des données de prolificité, nous avons négligé les fécondations obtenues après la lutte contrôlée, ce qui explique les différences d'effectifs entre les tableaux 5 et 6 relatifs à la fertilité et aux mises bas.

Les animaux contemporains des 2 races parentales sont toujours représentés, mais ils conservent leur rythme traditionnel de reproduction (lutte annuelle en juilletaoût, avec une première lutte à 18 mois), de sorte que la comparaison entre brebis croisées et parentales est possible à partir de 3 ans, mais discutable compte tenu de l'influence probable du début de carrière ( 3 agnelages à 3 ans pour les croisées contre 2 pour les brebis Berrichonnes et Romanov).

\section{Mode d'élevage des agneaux. Conduite des brebis}

Les agneaux sont élevés avec leur mère en bergerie de la naissance au sevrage et reçoivent une alimentation complémentaire (foin et concentré) dès l'âge d'un mois. Les agneaux en surnombre, appartenant à des portées multiples ou issus de mères à production laitière insuffisante sont élevés en allaitement artificiel au lait froid. Pour définir le type de naissance-allaitement, nous avons retenu 6 catégories [né et allaité simple $(1,1)$; né double allaité simple $(2,1)$; né double allaité double $(2,2)$; né multiple allaité simple ( 3 ou 4,1$)$; né multiple allaité double ou triple ( 3 ou 4,2 ou 3$)$; allaitement artificiel quel que soit le type de naissance (aa)] ou 5 catégories $[(1,1)$; ( 2 ou 3 ou 4,1$) ;(2,2)$; ( 3 ou 4,2 ou 3$)$, (aa)]. Les agnelles conservées pour la reproduction sont maintenues en bergerie depuis leur naissance jusqu'à 15 mois, c'est-à-dire jusqu'à la $1^{\text {re }}$ lutte d'avril. Elles vivent ensuite sur pâturage rationné jusqu'à la $1^{\text {re }}$ mise bas en septembre, puis rentrent en bergerie jusqu'en mai, c'est-à-dire jusqu'au sevrage de leurs agneaux nés de la $2^{\circ}$ mise bas. Elles sont ensuite regroupées avec le troupeau principal qui vit à l'extérieur d'avril à novembre.

Les 2 premiers agnelages sont peu surveillés : minimum d'interventions dans la journée et pas de ronde la nuit. A partir du $3^{\circ}$ agnelage, la surveillance des mises bas est plus régulière et les bergers interviennent si cela est nécessaire.

\section{Variables considérées}

La croissance des agnelles est mesurée par le poids à la naissance, 5 poids à âges standards $(10,30,50,70$ et 90 jours) calculés par intrapolation linéaire à partir des pesées effectuées toutes les 3 semaines après la naissance, et 2 gains moyens quotidiens (GMQ 10-30 jours et GMQ 30-70 jours). Les brebis sont pesées après chaque mise bas : il manque toutefois les poids à la mise bas 1 des brebis nées en 1973.

La production laitière est estimée d'après la somme des gains moyens quotidiens des agneaux allaités sous la mère (GMQ de 0 à 30 jours en $\mathrm{g}$ ), en éliminant les agneaux morts avant 15 jours et en tenant compte de la durée effective d'allaitement (durée de vie) pour ceux réformés du $15^{\circ}$ au $30^{\circ}$ jour. Cette estimation a été trans- 
formée en $\mathrm{kg}$ de lait, à partir de l'équation de RiCoRdeAu \& Boccard (1961) : kg de lait $=4,08$. GMQ + 449 .

Les performances de reproduction se résument à la fertilité aux différentes périodes de lutte, au nombre d'agneaux nés totaux (y compris les avortements), nés vivants et allaités par portée.

\section{Analyse statistique}

Compte tenu de la répartition des brebis par année de naissance et génération, nous avons complété l'analyse globale portant sur l'ensemble des données ( 5 années de naissance, génération F1 à F4), par des analyses séparées sur 3 blocs élémentaires (tabl. 1). Celles-ci donnent des résultats comparables à l'analyse globale, aussi nous les avons ignorées pour simplifier la présentation, sauf pour localiser certains effets plus ou moins significatifs. Les données ont été analysées par analyse de variance à effets fixés, en tenant compte des facteurs suivants :

- âge de la mère à la naissance des brebis ( 3 et 4 ans; 5 ans et plus) ou poids de la mère à la naissance des brebis (comme covariable) pour l'analyse de la croissance pondérale ;

- année de naissance des brebis (1973 à 1977);

- type de naissance-allaitement des brebis (6 catégories pour l'analyse de la croissance, 5 pour les autres variables).

Toutes les brebis ont été contrôlées jusqu'à 4 ans ( 4 mises bas), sauf celles nées en 1977 ( 3 ans et 3 mises bas). Les brebis F4 n'étant pratiquement pas représentées en $4^{\circ}$ mise bas, les comparaisons entre brebis des générations F1 à F4 doivent se limiter aux 3 premières mises bas. Cependant, comme les différences entre générations ne sont pas significatives, les valeurs enregistrées à la $4^{\circ}$ mise bas sur les brebis F1 à F3 donnent une indication assez précise de l'évolution des performances avec l'âge.

Ensuite, séparément pour les brebis des générations F2 et F3, nous avons estimé l'héritabilité et les corrélations phénotypiques et génétiques par covariance entre demi-sœurs de père, en utilisant un modèle considérant les effets année de naissance et type de naissance-allaitement comme fixés et l'effet père comme aléatoire.

\section{Résultats}

\section{Croissance des agnelles conservées pour la reproduction}

Les effets année de naissance et type de naissance-allaitement des jeunes brebis, de même que l'effet poids des mères à la mise bas, sont très significatifs sur toutes les variables (tabl. 3 a). Les agnelles nées simples ont les poids et les gains maximum, devant les agnelles nées doubles élevées simples, alors que les agnelles nées multiples et élevées doubles ou triples, ont les poids et les gains les plus faibles. Les agnelles élevées en allaitement artificiel, presque toutes nées multiples, se comportent de façon différente, puisqu'elles ont un très bon gain 10-30 jours (252 contre $249 \mathrm{~g}$ pour les allaités simples) mais un très mauvais gain $30-70$ jours (225 contre $280 \mathrm{~g}$ pour les allaités simples). On note cependant que l'interaction année $\times$ type de naissanceallaitement st très significative ou significative, sauf pour le gain 30-70 jours. 


\section{TABLEAU 3}

Moyennes ajustées des variables croissance des agnelles F1 à F4, en fonction de leur année de naissance, de leur type de naissance-allaitement et du poids de leurs mères à la mise bas.

Least-squares means of weights for ewe-lambs F1 to F4, according to their year of birth, their birth-rearing class and the lambing-weights of their mothers.

a) Analyse globale

\begin{tabular}{|c|c|c|c|c|c|c|c|c|c|}
\hline \multirow{2}{*}{ Facteur } & \multirow{2}{*}{$\mathbf{n}$} & \multicolumn{6}{|c|}{ Poids vif en $\mathrm{kg}$} & \multicolumn{2}{|c|}{ GMQ en $g / j$} \\
\hline & & nais. & $10 \mathrm{j}$ & $30 \mathrm{j}$ & $50 \mathrm{j}$ & $70 \mathrm{j}$ & $90 \mathrm{j}$ & $10-30 \mathrm{j}$ & $30-70 \mathrm{j}$ \\
\hline$\mu$ & 1298 & 3,55 & 5,4 & 9,6 & 14,4 & 19,6 & 24,8 & 209 & 253 \\
\hline Année de naissance & & TS & TS & TS & TS & TS & TS & TS & TS \\
\hline $1973 \ldots \ldots \ldots$ & 229 & 3,41 & 5,3 & 9,4 & 14,1 & 19,7 & 25,3 & 207 & 265 \\
\hline $1974 \ldots \ldots \ldots$ & 282 & 3,35 & 4,8 & 8,5 & 13,0 & 18,1 & 23,4 & 184 & 247 \\
\hline $1975 \ldots \ldots \ldots \ldots$ & 162 & 3,57 & 5,3 & 9,6 & 13,9 & 18,6 & 23,3 & 211 & 229 \\
\hline $1976 \ldots \ldots \ldots \ldots$ & 281 & 3,59 & 5,6 & 9,7 & 14,6 & 19,9 & 25,0 & 207 & 255 \\
\hline $1977 \ldots \ldots \ldots$ & 344 & 3,79 & 6,0 & 10,6 & 15,8 & 21,0 & 26,2 & 233 & 260 \\
\hline $\begin{array}{l}\text { Type naissance-al- } \\
\text { laitement } \ldots . . . \ldots\end{array}$ & & & TS & TS & ${ }^{\mathrm{T}} \mathrm{TS}$ & TS & TS & TS & TS \\
\hline $1,1 \ldots \ldots \ldots \ldots$ & 121 & 4,42 & 6,8 & 11,7 & 17,3 & 22,9 & 28,6 & 249 & 280 \\
\hline $2,1, \ldots \ldots \ldots$ & 93 & 3,71 & 5,9 & 10,5 & 15,6 & 21,1 & 26,5 & 229 & 267 \\
\hline $2,2 \ldots \ldots \ldots \ldots$ & 681 & 3,65 & 5,3 & 9,2 & 13,9 & 19,3 & 24,5 & 192 & 256 \\
\hline 3 ou $4,1 \ldots \ldots$ & 21 & 3,31 & 5,0 & 9,3 & 14,1 & 19,2 & 24,2 & 218 & 247 \\
\hline 3 ou 4,2 ou 3 & 173 & 3,07 & 4,7 & 8,5 & 13,0 & 18,2 & 23,5 & 186 & 249 \\
\hline a. a. $\ldots \ldots \ldots$ & 209 & 3,08 & 5,4 & 10,4 & 14,9 & 19,3 & 23,9 & 252 & 225 \\
\hline $\mathrm{N}^{\circ}$ de génération & & $\mathrm{TS}$ & TS & TS & TS & $S$ & $S$ & TS & NS \\
\hline$F 1 \quad \ldots \ldots \ldots \ldots$ & 200 & 3,36 & 5,1 & 9,2 & 13,8 & 19,0 & 24,1 & 202 & 248 \\
\hline F2 $\ldots \ldots \ldots \ldots$ & 530 & 3,68 & 5,6 & 9,8 & 14,6 & 19,8 & 25,0 & 210 & 254 \\
\hline$\ldots \ldots \ldots$ & 478 & 3,46 & 5,3 & 9,5 & 14,3 & 19,5 & 24,7 & 209 & 254 \\
\hline F4 $\ldots \ldots \ldots$ & $90^{-}$ & 3,75 & 5,8 & 10,3 & 15,2 & 20,4 & 25,6 & 226 & 255 \\
\hline \multicolumn{10}{|l|}{ Interactions } \\
\hline Année $\times$ Type $N$ & A. $\cdots$ & & TS & TS & TS & $\mathbf{S}$ & $\mathbf{S}$ & TS & NS \\
\hline Année $\times \mathrm{N}^{\circ}$ gén & ération & TS & TS & TS & TS & TS & TS & TS & TS \\
\hline Poids de la mère & & $\mathrm{TS}$ & TS & TS & TS & TS & TS & TS & TS \\
\hline$b_{i} \ldots \ldots \ldots$ & & 0,014 & 0,021 & 0,037 & 0,049 & 0,059 & 0,065 & 0,68 & 0,50 \\
\hline
\end{tabular}

b) Analyse par bloc : Effet $n^{\circ}$ de génération

\begin{tabular}{|c|c|c|c|c|c|c|c|c|}
\hline 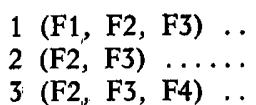 & $\begin{array}{r}511 \\
1008 \\
595\end{array}$ & $\begin{array}{l}\text { TS } \\
\text { TS } \\
\text { NS }\end{array}$ & $\begin{array}{l}\text { TS } \\
\text { S } \\
\text { NS }\end{array}$ & $\begin{array}{l}\text { TS } \\
\text { NS } \\
\text { NS }\end{array}$ & $\begin{array}{l}\text { TS } \\
\text { NS } \\
\text { NS }\end{array}$ & $\begin{array}{l}\text { TS } \\
\text { NS } \\
\text { NS }\end{array}$ & $\begin{array}{c}\text { TS } \\
\text { NS } \\
\text { S }\end{array}$ & $\begin{array}{l}\text { S } \\
\text { NS } \\
\text { NS }\end{array}$ \\
\hline
\end{tabular}

TS $=$ très significatif $(p<0,01) ; S=$ significatif $(p<0,05) ; N S=$ non significatif. 


\section{TABleau 4}

Moyennes ajustées des poids vifs des brebis après les 4 premières mises bas, en fonction de leur année de naissance, de leur propre type de naissance-allaitement et de l'âge de leur mère.

Least-squares means of lambing-weight of the ewes after the first-four lambings, according to their year of birth, their own birth-rearing class and the age of their dam.

\begin{tabular}{|c|c|c|c|c|c|c|c|c|}
\hline \multirow[b]{2}{*}{ Facteurs } & \multicolumn{2}{|c|}{ Mise bas 1} & \multicolumn{2}{|c|}{ Mise bas 2} & \multicolumn{2}{|c|}{ Mise bas 3} & \multicolumn{2}{|c|}{ Mise bas 4} \\
\hline & $\mathbf{n}$ & $\begin{array}{l}\text { P.V. } \\
\text { en } \mathbf{k g}\end{array}$ & $\mathrm{n}$ & $\begin{array}{l}\text { P.V. } \\
\text { en kg }\end{array}$ & $\mathbf{n}$ & $\begin{array}{l}\text { P.V. } \\
\text { en } \mathbf{~ k g}\end{array}$ & $\mathbf{n}$ & $\begin{array}{l}\text { P.V. } \\
\text { en kg }\end{array}$ \\
\hline $\boldsymbol{\mu}$ & 929 & 54,5 & 889 & 57,0 & 761 & 62,8 & 503 & 65,6 \\
\hline Age des mères $\ldots . .$. & & NS & & $\mathrm{S}$ & & NS & & NS \\
\hline 3 et 4 ans $\ldots \ldots \ldots$ & 694 & 54,4 & 647 & 56,6 & 559 & 62,5 & 372 & 65,1 \\
\hline 5 ans et plus $\ldots \ldots$ & 235 & 54,9 & 242 & 58,0 & 202 & 63,6 & 131 & 67,2 \\
\hline $\begin{array}{l}\text { Année de naissance des } \\
\text { brebis } \ldots \ldots \ldots \ldots \ldots \ldots\end{array}$ & & TS & & TS & & TS & & TS \\
\hline $1973 \ldots \ldots \ldots \ldots$ & 0 & - & 177 & 58,1 & 167 & 60,4 & 146 & 66,3 \\
\hline $1974 \ldots \ldots \ldots \ldots \ldots$ & 243 & 51,8 & 179 & 54,9 & 205 & 62,5 & 149 & 59,7 \\
\hline $1975 \quad \ldots \ldots \ldots \ldots \ldots$ & 146 & 56,2 & 105 & 61,5 & 110 & 57,6 & 79 & 66,3 \\
\hline $1976 \ldots \ldots \ldots \ldots$ & 237 & 53,8 & 189 & 55,3 & 111 & 65,7 & 129 & 71,2 \\
\hline $1977 \ldots \ldots \ldots \ldots$ & 303 & 56,3 & 239 & 57,1 & 168 & 67,1 & - & - \\
\hline $\begin{array}{l}\text { Type Naiss.-allaitement } \\
\text { des brebis } \ldots \ldots \ldots \ldots\end{array}$ & & TS & & TS & & $\mathbf{S}$ & & $\mathbf{S}$ \\
\hline $1 ; 1 \ldots \ldots \ldots \ldots$ & 88 & 55,4 & 80 & 58,8 & 67 & 64,2 & 50 & 67,7 \\
\hline 2,3 ou $4 ; 1$. & 88 & 55,8 & 77 & 57,5 & 62 & 63,8 & 29 & 67,3 \\
\hline $2 ; 2 \ldots \ldots \ldots$ & 535 & 54,7 & 461 & 57,3 & 385 & 63,1 & 253 & 65,9 \\
\hline 3 ou $4 ; 2$ ou $3 \ldots$ & 86 & 53,0 & 111 & 55,5 & 116 & 61,0 & 82 & 64,5 \\
\hline a. a. $\ldots \ldots \ldots \ldots$ & 132 & 53,2 & 160 & 56,0 & 131 & 62,3 & 89 & 64,0 \\
\hline $\mathrm{N}^{\circ}$ de génération $\ldots$. & & NS & & NS & & NS & & NS \\
\hline F1 $\ldots \ldots \ldots \ldots \ldots$ & 42 & 55,5 & 145 & 57,5 & 140 & 62,6 & 119 & 66,7 \\
\hline F2 $\ldots \ldots \ldots \ldots \ldots$ & 393 & 54,2 & 372 & 56,4 & 303 & 62,5 & 210 & 65,0 \\
\hline F3 $\ldots \ldots \ldots \ldots \ldots$ & 414 & 54,5 & 309 & 57,0 & 275 & 62,9 & 164 & 65,6 \\
\hline F4 $\ldots \ldots \ldots \ldots \ldots$ & 80 & 55,2 & 63 & 59,2 & 43 & 64,9 & - & $\longrightarrow$ \\
\hline Interactions & & & & & & & & \\
\hline Année $\times$ Type N.A. & & NS & & NS & & NS & & NS \\
\hline Année $\times \mathrm{N}^{\circ}$ génér. & & NS & & NS & & NS & & NS \\
\hline
\end{tabular}

Les brebis nées en 1973 n'ont pas été pesées à la première mise bas.

La prise en compte, dans ce modèle, des 2 types de $\mathrm{F} 1[\mathrm{~F} 1$ (père $\mathrm{RO} \times$ mère $\mathrm{BC}$ ) et $\mathrm{F} 1$ (père $\mathrm{BC}$ $\times$ mère RO)] ne fait apparaître aucune différence dans les poids aux mises bas 2 à 4 : respectivement 56,8 et 57,$6 ; 63,6$ et 62,$4 ; 66,5$ et $66,7 \mathrm{~kg}$. 

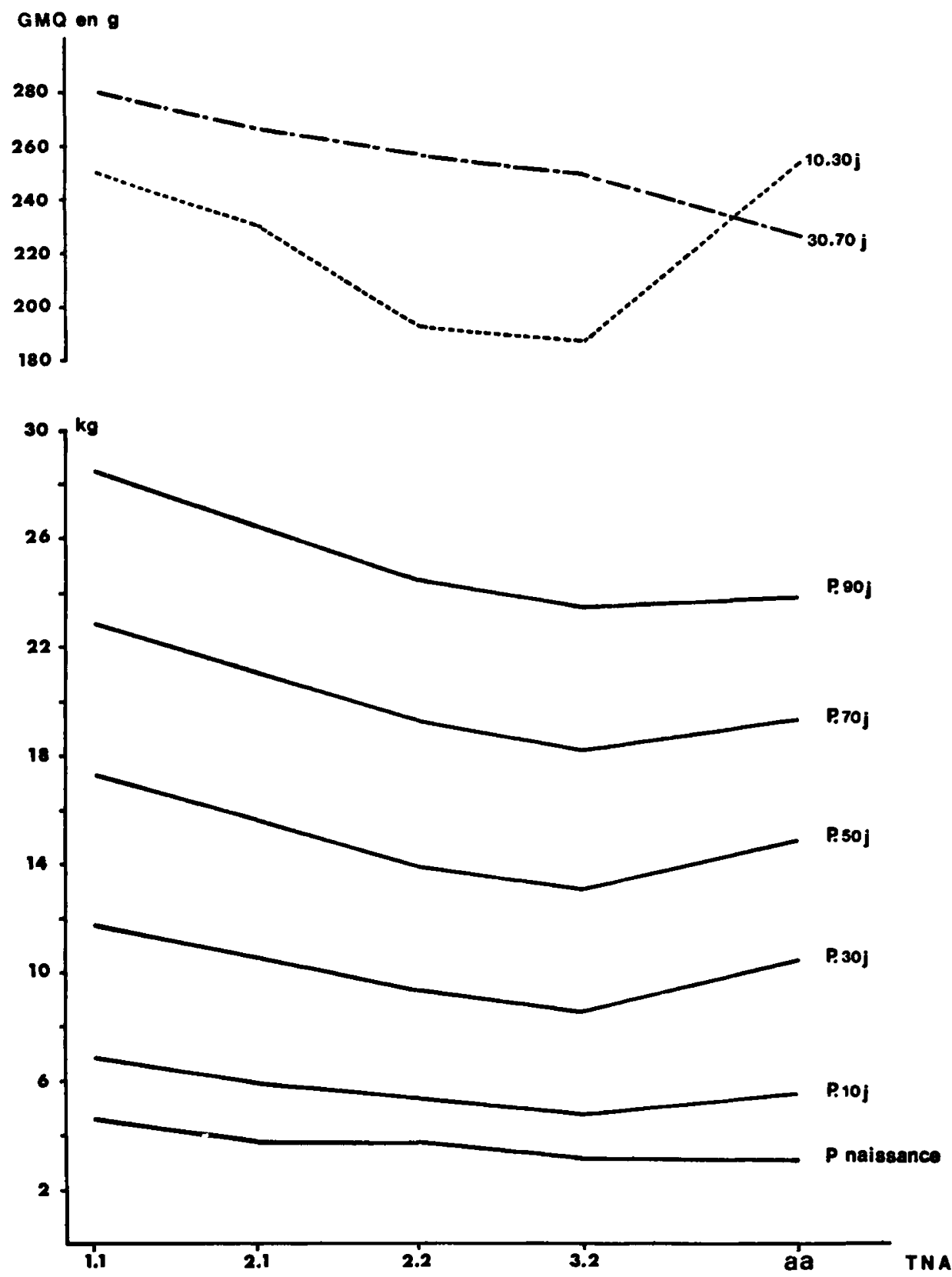

FIG. 1

Croissance des agnelles $F 1$ d F4 de la naissance d 90 jours suivant leur type de naissance-allaitement.

Growth traits of ewe-lambs F1 to F4, from birth to 90 days, according to their birth-rearing class. 
Les différences entre générations sont très significatives jusqu'à 50 jours, significatives pour les poids à 70 et 90 jours et non significatives pour le gain 30-70 jours. Elles s'expliquent par les performances inférieures des agnelles $F 1$ nées des 2 races parentales, en dépit de la prise en compte du poids des mères. En effet, la comparaison des analyses par bloc (tabl. $3 \mathrm{~b}$ ) montre que les différences entre générations sont toujours significatives dans le bloc 1 qui inclut les F1, mais jamais significatives au-delà de 30 jours dans les blocs 2 et 3 ne comprenant pas de F1.

\section{Poids d̀ la mise bas}

Le poids à la mise bas augmente de 54,5 à $65,6 \mathrm{~kg}$ de la $1^{\text {re }}$ à la $4^{\text {e }}$ mise bas, soit un accroissement de 20,4 p. 100 (tabl. 4). Les brebis nées de mères âgées ont tendance à être plus lourdes, mais l'effet de l'âge des mères n'est significatif qu'à la $2^{\circ}$ mise bas. Les différences entre années de naissance des brebis sont toutes très significatives. Ce qui est plus inattendu, c'est l'influence prolongée du type de naissance-allaitement des brebis sur leur propre poids de 1 à 4 ans : effet très significatif aux mises bas 1 et 2 , significatif aux mises bas 3 et 4 , et sans interaction année $X$ type de naissance-allaitement. Ainsi, à la $2^{\circ}$ mise bas, les brebis nées et allaitées simples ont, par rapport aux brebis nées et allaitées doubles d'une part, nées multiples et allaitées doubles ou triples d'autre part, un avantage de 1,5 et $3,3 \mathrm{~kg}$, alors que cet écart était de 4,1 et $5,1 \mathrm{~kg}$ à 3 mois. Les brebis allaitées artificiellement ont des poids comparables à celles nées et allaitées multiples, et les brebis nées multiples mais allaitées simples ont des poids comparables à celles nées et allaitées simples. Finalement, le classement en poids vif acquis à 70 ou 90 jours se conserve jusqu'à 4 ans.

Les différences entre générations ne sont pas significatives. On note également que les brebis $\mathrm{F} 1$ nées de mères Romanov ont dès la $2^{\circ}$ mise bas un poids comparable à leurs contemporaines nées de mères Berrichonne.

\section{Fertilité}

Pour les brebis nées la même année, l'effet période de lutte est très significatif : la fertilité est de 0,86 à la $1^{\text {re }}$ lutte de mai, de 0,82 à la $2^{\circ}$ lutte post-partum et atteint 0,97-0,99 à partir de la $3^{\circ}$ lutte (tabl. 5). Cependant, intra-année de naissance et période, les différences entre générations ne sont pas significatives.

La durée de la $1^{\text {re }}$ lutte est d'environ 2 cycles. La distribution des saillies fécondantes estimée indirectement d'après les dates de mises bas est assez variable d'une année à l'autre. On observe bien certaines années des * pics» de fécondation 12 à 19 ou 28 à 40 jours après l'introduction des béliers vasectomisés, mais ces pics ne sont pas répétables et ne traduisent aucun \& effet bélier * marqué.

La fertilité est légèrement plus faible à la $2^{\text {e }}$ lutte post-partum en saison, car la période effective des saillies est limitée, notamment dans le cas où la première mise bas est tardive. A titre indicatif, en septembre-octobre 1978 , l'intervalle $1^{\text {re }}$ mise bas fécondation est en moyenne de 44 jours, avec 82 p. 100 des fécondations de 32 à 61 jours post-partum. 


\section{Tableau 5}

Fertilité des brebis : moyennes ajustées (p. 100) par numéro de mise bas, intra-année de naissance.

Fertility of ewes : least squares means (p. 100) at different matings, within year of birth.

\begin{tabular}{|c|c|c|c|c|c|}
\hline \multirow[b]{2}{*}{ Année de naissance } & \multicolumn{5}{|c|}{ Périodes de lutte et âge } \\
\hline & 16 mois & $\stackrel{2}{22}$ mois & $\begin{array}{l}3 \\
31 \text { mois }\end{array}$ & $\begin{array}{l}4 \\
43 \text { mois }\end{array}$ & $\begin{aligned} & 5 \text { à } 7 \\
> & 55 \text { mois }\end{aligned}$ \\
\hline $1973 \ldots \ldots \ldots \ldots \ldots$ & 80,5 & 83,1 & 96,8 & 97,6 & 98,5 \\
\hline $1974 \ldots \ldots \ldots \ldots \ldots$ & 84,0 & 78,5 & 97,7 & 97,0 & 98,2 \\
\hline $1975 \ldots \ldots \ldots \ldots \ldots$ & 94,6 & 78,7 & 97,5 & 100,0 & 100,0 \\
\hline $1976 \ldots$ & 85,1 & 84,7 & 94,6 & 97,6 & - \\
\hline $1977 \ldots \ldots \ldots \ldots \ldots$ & 86,9 & 83,9 & 97,8 & 一 & 一 \\
\hline Moyenne arithmétique & 86,2 & 81,8 & 96,9 & 98,0 & 99,0 \\
\hline n $\ldots$ & 1438 & 1221 & 945 & 557 & 570 \\
\hline
\end{tabular}

\section{Prolificité}

\subsection{Nés totaux}

La prolificité est de $1,67,1,87,1,98$ et 2,01 aux mises bas 1 à 4 (tabl. 6), ce qui correspond à une augmentation de 20,4 p. 100. L'effet âge des mères n'est pas significatif. L'effet année de naissance n'est significatif qu'à la $1^{\text {re }}$ mise bas. L'effet type de naissance-allaitement des brebis (en tant qu'agnelles) est très significatif à la mise bas 1 et non significatif ensuite : les antenaises nées simples ont une prolificité supérieure à celles nées multiples $(+0,05$ à $+0,12)$, mais ce sont les antenaises élevées en allaitement artificiel qui ont la prolificité maximum. Les différences entre générations ne sont jamais significatives, de même que les interactions "année $\times \mathrm{n}^{\circ}$ de génération » et "année $\times$ type de naissance-allaitement ». Le tableau 7 indique, pour les trois premiers agnelages, la répartition des tailles de portée et le pourcentage d'agneaux en allaitement artificiel : respectivement 38,31 et 16 p. 100 de simples; 58,53 et 69 p. 100 de doubles; 4,15 et 15 p. 100 de triples ; 8, 14 et 12 p. 100 d'agneaux en allaitement artificiel.

\subsection{Nés vivants}

Les effets des facteurs de variation sont pratiquement identiques à ceux observés pour les nés totaux (tabl. 6). A.la mise bas 1, les antenaises nées simples ont un nombre de nés vivants supérieur à celles nées multiples $(+0,10$ à 0,15$)$ et ce sont toujours les antenaises élevées en allaitement artificiel qui ont le maximum de nés vivants. 


\begin{tabular}{|c|c|c|c|c|c|c|c|c|}
\hline & 気 & $\begin{array}{l}> \\
\mathscr{\Xi} \\
\Xi\end{array}$ & $\stackrel{\infty}{\infty}$ & 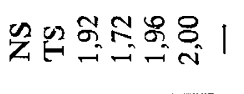 & 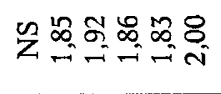 & 饥总志志 & 㕣公 & 言 \\
\hline & 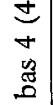 & $\begin{array}{l}H \\
\mathscr{m} \\
=\end{array}$ & ¿ & 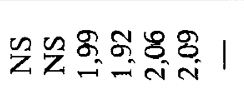 & 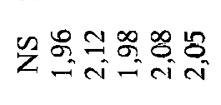 & 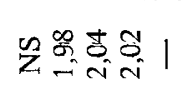 & 舟兄 & $\begin{array}{l}\text { 芯 } \\
\text { 芯 } \\
\text { 芯 }\end{array}$ \\
\hline$\stackrel{\dot{0}}{\frac{0}{2}}$ & $\stackrel{\mathscr{D}}{\stackrel{2}{\Sigma}}$ & $\approx$ & $\overrightarrow{\text { in }}$ & $\stackrel{\infty}{ \pm} \overrightarrow{0}$ & 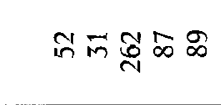 & 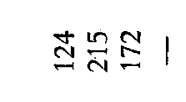 & & 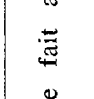 \\
\hline 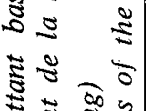 & $\widehat{\overparen{\Xi}}$ & $\begin{array}{l}p \\
\ddot{z}\end{array}$ & $\infty$ & 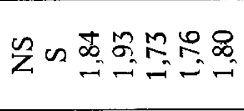 & 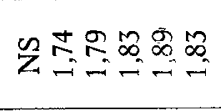 & 乙悉冓市 & $\mathscr{n} \tilde{Z}$ & $\widetilde{0}_{-\infty}^{+\infty}$ \\
\hline 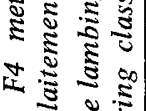 & 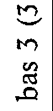 & $\begin{array}{l}F \\
\ddot{z}\end{array}$ & $\stackrel{g}{-}$ & 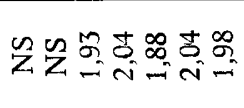 & 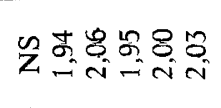 & 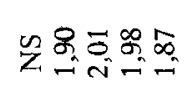 & 公兄 & 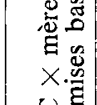 \\
\hline 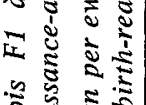 & $\sum_{\sum}^{\stackrel{n}{2}}$ & $=$ & 㣽 & 䟲气さ怘 & ゚シき昌 & 昌胥麗 & & 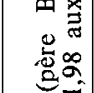 \\
\hline 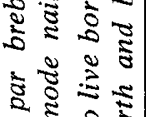 & $N$ & $\begin{array}{l}> \\
\text { 量 } \\
\end{array}$ & in & 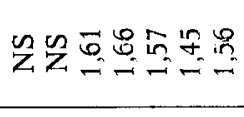 & 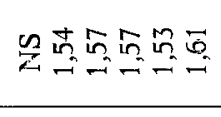 & 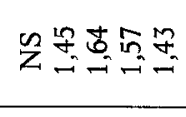 & 㔯咨 & 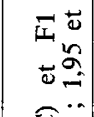 \\
\hline 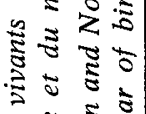 & 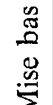 & $\begin{array}{l}H \\
\ddot{g}\end{array}$ & $\stackrel{\infty}{-\infty}$ & 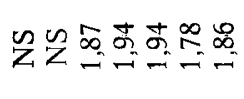 & 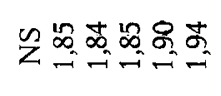 & 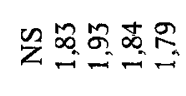 & z & 造通 \\
\hline 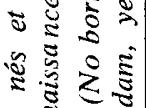 & & $=$ & 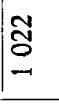 & 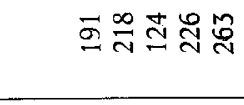 & 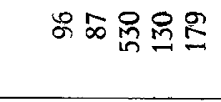 & 톸웜뮴요 & & a \\
\hline 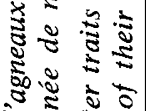 & - & 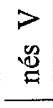 & $\stackrel{n}{2}$ & 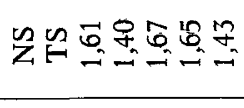 & 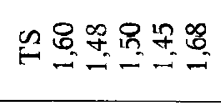 & 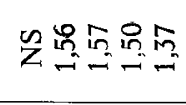 & 象兄 & 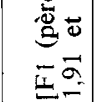 \\
\hline 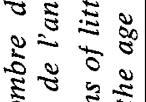 & 总 & 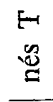 & $\hat{\sigma}_{-}$ & 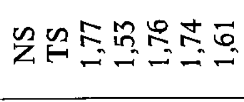 & 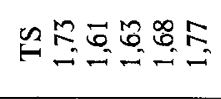 & 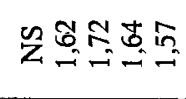 & $\tilde{z} \tilde{z}$ & $\frac{n}{2}$ \\
\hline 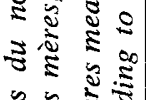 & & $=$ & g & 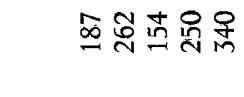 & 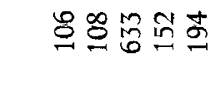 & 츤 & & 过 \\
\hline 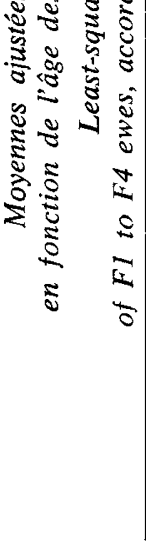 & & 吾 & 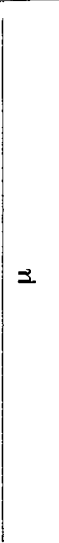 & 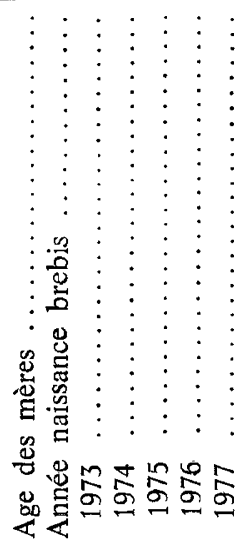 & 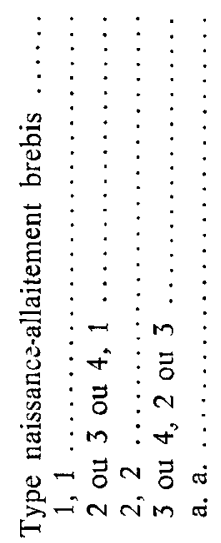 & 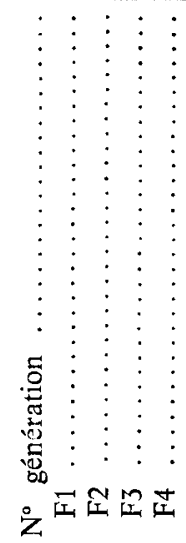 & 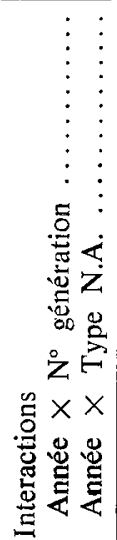 & 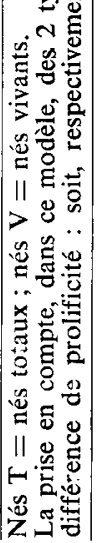 \\
\hline
\end{tabular}




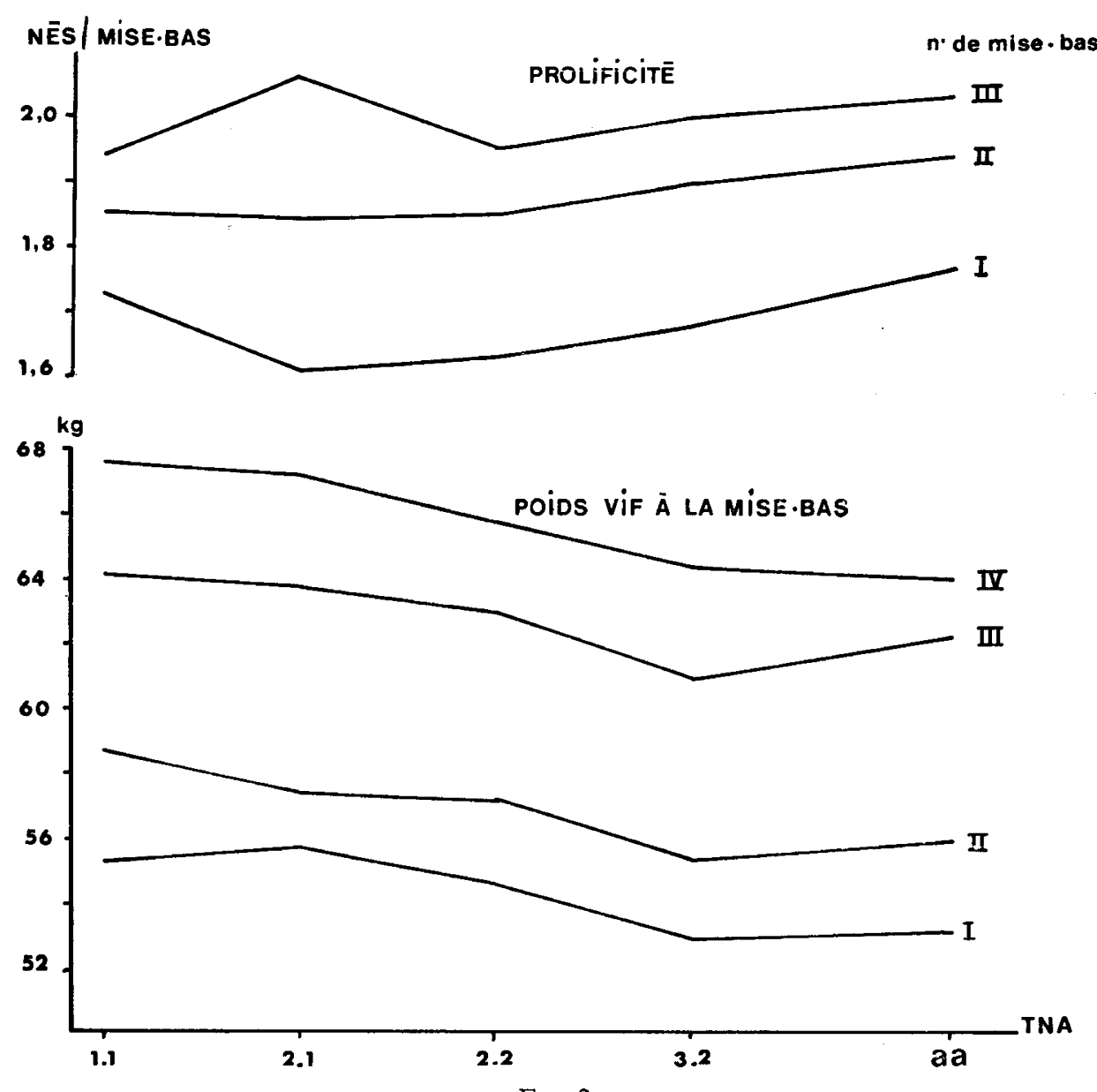

FIG. 2

Poids vif et prolificité des brebis F1 à F4, suivant leur type de naissance-allaitement.

Weight and litter size of F1 to F4 ewes, according to their own birth-rearing class.

\section{TABLEaU 7}

Répartition (p. 100) des tailles de portée des brebis $F 1$ à $F 4$ et proportion d'agneaux en allaitement artificiel, aux 3 premières mises bas.

Distribution ( $p .100$ ) of litter size of F1 to F4 ewes and proportion of fostered lambs at the first-three lambings.

\begin{tabular}{|c|c|c|c|}
\hline Taille de portée & Mise bas 1 & Mise bas 2 & Mise bas 3 \\
\hline $\begin{array}{l}1 \\
2 \\
2 \\
3 \\
3 \\
4 \\
4\end{array}$ & $\begin{array}{r}38,2 \\
58,0 \\
3,8 \\
-\end{array}$ & $\begin{array}{r}31,0 \\
52,9 \\
15,4 \\
0,7\end{array}$ & $\begin{array}{l}16,5 \\
68,9 \\
14,6 \\
-\end{array}$ \\
\hline $\begin{array}{l}\text { Agneaux en allaitement artifi- } \\
\text { ciel }(*) \quad \ldots \ldots \ldots \ldots \ldots \ldots\end{array}$ & 7,7 & 14,1 & 12,4 \\
\hline (*) En p. 100 des nés vivants. & & & \\
\hline
\end{tabular}




\subsection{La mortalité des agneaux à la naissance (avortements compris)}

Elle est de $8,3,16,0,7,6$ et 7,0 p. 100 aux mises bas 1 à 4 . Ces différences traduisent un effet de l'âge des brebis et surtout de la période d'agnelage : en effet, les deux premiers agnelages sont peu ou pas surveillés et le $2^{\circ}$ agnelage, consécutif à une gestation effectuée entièrement en bergerie, succède à l'agnelage principal du reste du troupeau.

\subsection{La prolificité dépend peu du poids à la mise bas}

Le coefficient de régression linéaire de la taille de portée sur le poids correspondant en $\mathrm{kg}$ est uniquement significatif à la mise bas $1\left(b_{i}=0,007\right)$.

\section{Production laitière}

\subsection{Nombre d'agneaux allaités}

Cette variable n'a qu'une valeur indicative, car elle dépend du nombre d'agneaux nés vivants, mais aussi du berger qui apprécie l'aptitude maternelle et la capacité laitière des brebis. Le nombre d'allaités varie de 1,47 à 1,67 de la $1^{\text {re }}$ à la $4^{\mathrm{e}}$ mise bas (tabl. 8). L'effet de l'année de naissance est significatif lors des mises bas 1 et 2 . Tous les autres effets sont non significatifs, notamment le $\mathrm{n}^{\circ}$ de génération.

\subsection{Quantité de lait consommée par les agneaux}

Elle est estimée après prise en compte du nombre d'agneaux nés et allaités comme covariable (tabl. 8). L'effet année de naissance est très significatif sauf en mise bas 2 . Les effets âge de la mère, type de naissance-allaitement des brebis sont non significatifs. L'effet $\mathrm{n}^{\circ}$ de génération n'est pas significatif, de même que l'interaction «année $\times \mathrm{n}^{\circ}$ de génération ». Exprimée en $\mathrm{kg}$ de lait, la production laitière journalière augmente régulièrement de la $1^{\text {re }}$ à la $4^{\circ}$ mise bas : respectivement $1,53,1,66$, 1,95 et $2,01 \mathrm{~kg}$, ce qui représente un accroissement de $31,4 \mathrm{p}$. 100 . Si les coefficients de régression de la production laitière estimée sur le nombre d'agneaux allaités sont nettement positifs $(+119$ à $+149 \mathrm{~g}$ de gain), ils sont légèrement négatifs $(-22$ à - $17 \mathrm{~g}$ de gain) sur le nombre d'agneaux nés : en effet, les agneaux nés multiples ont un poids de naissance plus faible et consomment moins de lait que les simples au cours de la phase initiale de croissance.

\section{Comparaison avec les brebis des 2 races parentales}

Nous avons limité (tabl. 9) la comparaison aux brebis Berrichonne et Romanov contemporaines des brebis croisées F2 à F4 nées en 1976 et 1977 (bloc 3), en ce qui concerne le poids à la mise bas et la prolificité à 3 et 4 ans, étant entendu qu'à cet âge, les brebis parentales ont réalisé un agnelage de moins que les croisées. Les performances des croisées sont peu différentes des moyennes parentales, aussi bien pour les poids vifs $(+1,0$ et $+1,6 \mathrm{~kg}$ à 3 et 4 ans) que pour le nombre de nés totaux $(-0,08$ et $+0,02)$. 


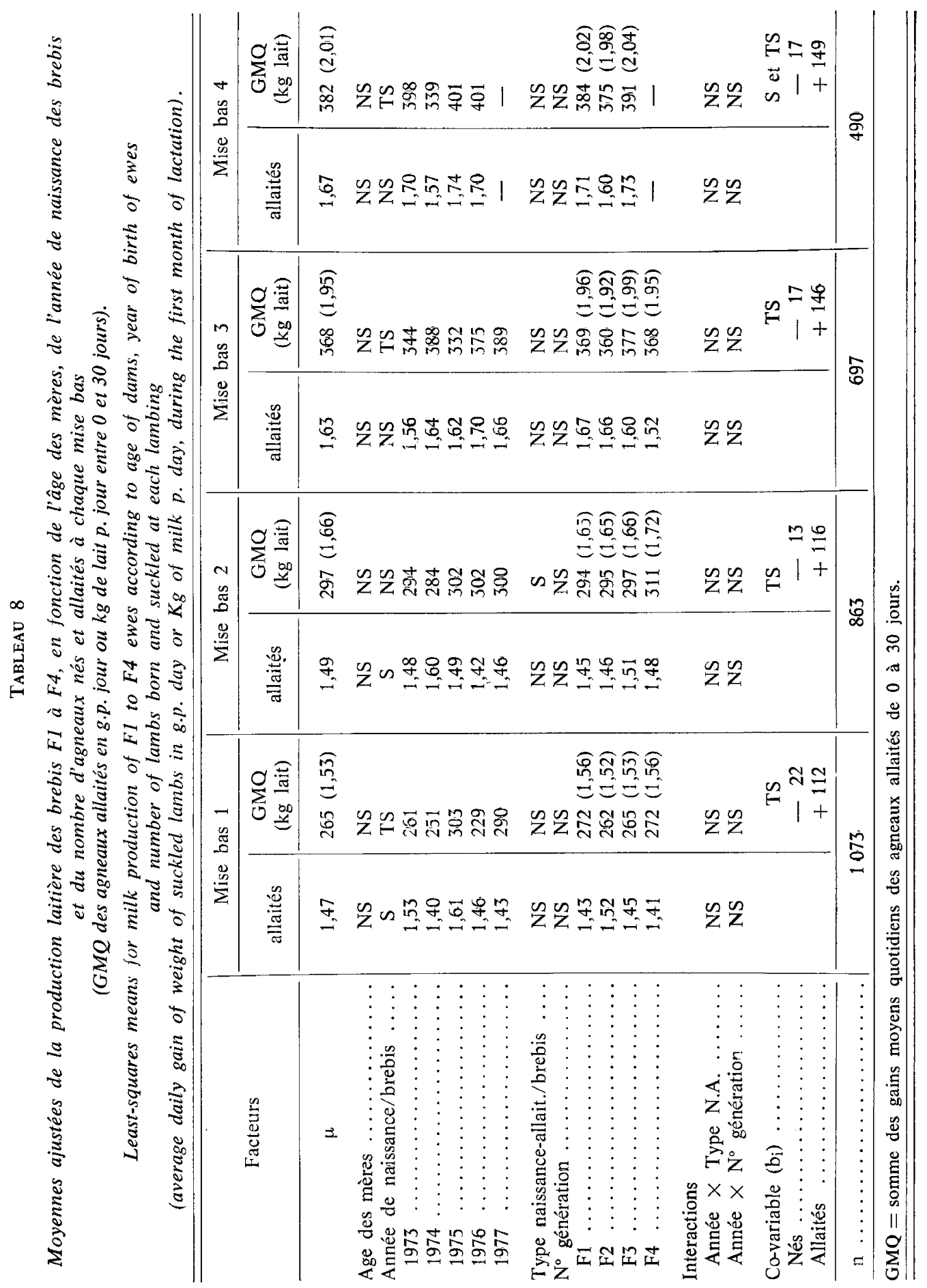


TABleau 9

Comparaison des brebis croisées $F 2$ à $F 4$ aux 2 races parentalcs : poids à la mise bas et taille de portée à 3 et 4 ans.

Comparison of crossbred F2 to F4 ewes to the two parental breeds : weight at lambing and litter size at 3 and 4 years.

\begin{tabular}{|c|c|c|c|c|c|}
\hline \multirow{2}{*}{$\begin{array}{l}\text { Année } \\
\text { de naissance }\end{array}$} & \multirow{2}{*}{$\begin{array}{c}\text { Type } \\
\text { génétique }\end{array}$} & \multicolumn{2}{|c|}{ Poids vif $(\mathrm{kg})$} & \multicolumn{2}{|c|}{ Nés totaux } \\
\hline & & 3 ans & 4 ans & 3 ans & 4 ans \\
\hline \multirow{4}{*}{1976} & Berrichon & $73,5(64)$ & $72,1 \quad(46)$ & 1,45 & 1,39 \\
\hline & Romanov & $58,0 \quad(39)$ & $56,0 \quad(52)$ & 2,72 & 2,69 \\
\hline & Croisées & 65,8 & 65,9 & 2,06 & 2,07 \\
\hline & $F_{i}-\bar{x}_{p}$ & 0 & $+1,8$ & $-0,03$ & $+0,03$ \\
\hline \multirow{4}{*}{1977} & Berrichon & $74,0 \quad(53)$ & $79,1 \quad(50)$ & 1,43 & 1,48 \\
\hline & Romanov & $57,1 \quad(84)$ & $60,7(25)$ & 2,82 & 2,72 \\
\hline & Croisées & 67,5 & 71,2 & 2,00 & 2,11 \\
\hline & $F_{i}-\bar{x}_{p}$ & $+1,9$ & $+1,3$ & $-0,13$ & $+0,01$ \\
\hline
\end{tabular}

$F_{\mathrm{i}}-\overline{\mathbf{x}}_{\mathrm{p}}=$ écart entre croisées et moyenne parentale.

3 ans $=2^{\mathrm{e}}$ mise bas pour les 2 races parentales et $3^{\mathrm{e}}$ mise bas pour les croisées.

4 ans $=3^{e}$ mise bas pour les 2 races parentales et $4^{e}$ mise bas pour les croisées.

7. Paramètres génétiques (tabl. 10)

Le gain 30-70 jours à une héritabilité significative ou très significative en F2 et F3 $(+0,17$ et $+0,43)$. L'héritabilité de la production de lait n'est jamais significative : 0,07 à 0,16 en F3. L'héritabilité de la prolificité à la $1^{\text {re }}$ mise bas est de 0,09 et 0,24 en $\mathrm{F} 2$ et $\mathrm{F} 3$; elle est très significative uniquement en $\mathrm{F} 3$ à la $2^{\circ}$ mise bas.

Entre les performances de croissance des jeunes agnelles et leur prolificité à la première mise bas, les corrélations phénotypiques sont légèrement positives ou nulles ( $-0,03$ pour le gain $30-70$ jours à $+0,30$ pour le gain $10-30$ jours); en revanche, les corrélations génétiques sont négatives avec les variables concernant le $1^{\mathrm{cr}}$ mois de croissance, mais nettement positives avec les poids à 70 et 90 jours $(+0,15$ à $+0,29)$ et surtout le gain 30-70 jours $(+0,41$ et $+0,36$ en F2 et F3). La corrélation génétique entre prolificité et production laitière est nettement positive en $F 3(+0,44)$, mais il s'agit là d'une valeur surestimée (car essentiellement phénotypique) puisque la production laitière n'a pas été corrigée pour le nombre d'agneaux nés et allaités. 


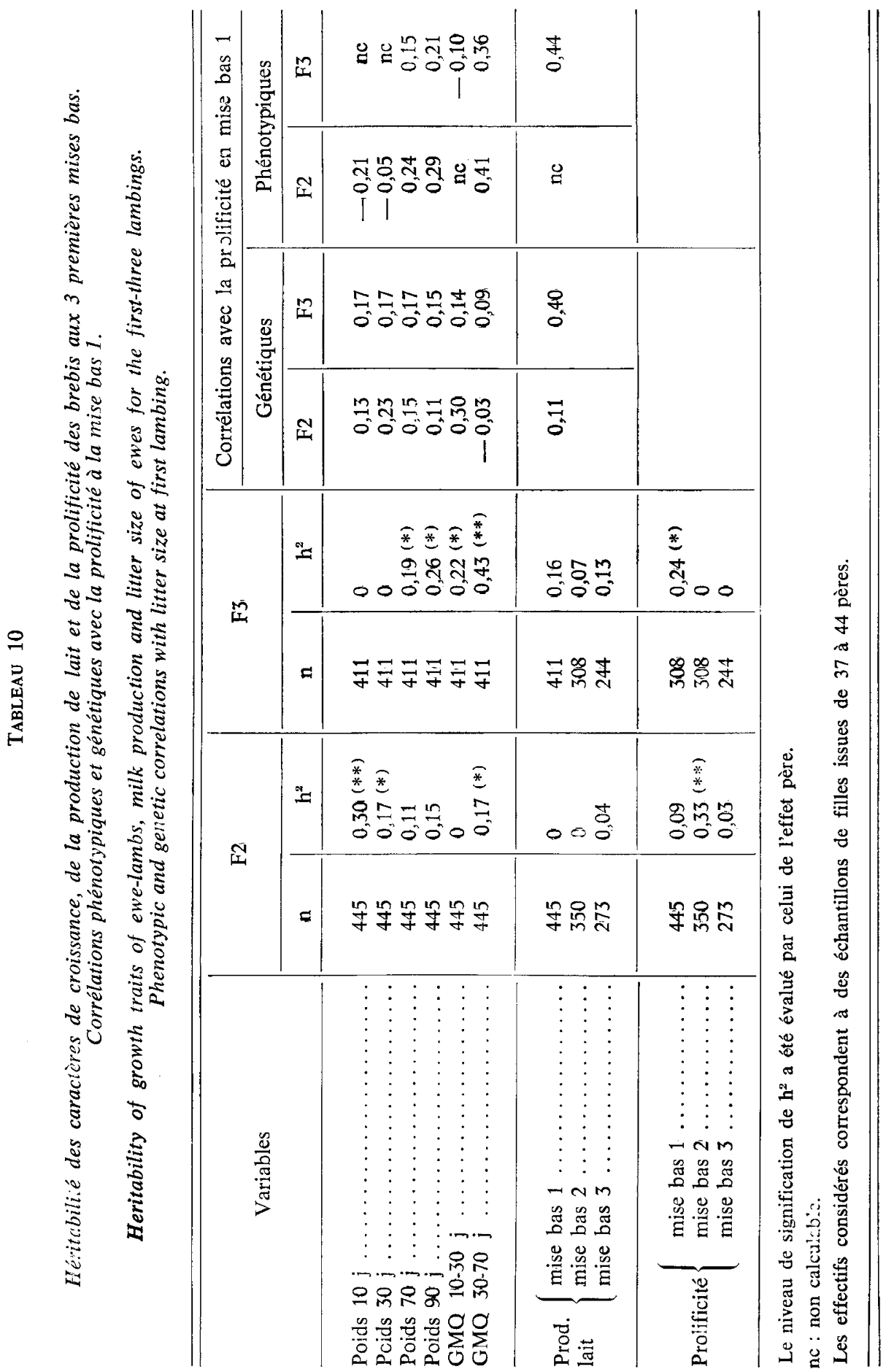




\section{Discussion}

\section{Différences entre générations}

Nous avons vu que ces différences sont significatives pour les variables croissance - sauf le gain 30-70 jours - mais non significatives pour le poids à la mise bas et les variables «aptitudes maternelles» comme la fertilité, le nombre des nés totaux et vivants par portée et la production laitière. Nous avons également vérifié qu'il n'existe pas de différence significative entre brebis F2, F3 et F4 en ce qui concerne le taux de réussite à la $1^{\text {re }}$ saillie lors des luttes 3 et 4 , ce qui laisse supposer que la mortalité embryonnaire n'augmente pas. Ces résultats confirment donc les premières observations enregistrées sur les brebis F1 et F2 nées de 1970 à 1972 et soumises à un rythme annuel de reproduction : hétérosis inférieur à 4 p. 100 et pas d'effet maternel sur la prolificité des F1 issues des accouplements réciproques; pas de différence entre $F 1$ et $F 2$ en ce qui concerne l'activité sexuelle, le taux d'ovulation et la mortalité embryonnaire (Ricordeau et al., $1976 \mathrm{a}, \mathrm{b}, \mathrm{c}, \mathrm{d}$ ). L'absence de «régression» des performances de reproduction de la F1 à la F4, traduirait donc l'absence d'hétérosis sur les effets génétiques directs et maternels et l'absence de «pertes » dues aux recombinaisons épistatiques, si l'on se réfère au modèle de DiCKERSON (1969).

C'est là que réside l'intérêt essentiel de cette étude. En effet, dans toutes les expérimentations de croisement réalisées à partir des races prolifiques Finnoise (Irlande : Hanrahan, 1974 ; U.S.A. : Oltenacu \& Boylan, 1981 a et b) ou Border Leicester (Australie : Pattie \& Smith, 1964 ; Nouvelle-Zélande : Clarke, 1962 ; Hight et al., 1973 ; Coop, 1974), on enregistre une diminution de la fertilité et de la prolificité de la F1 à la F2, ou même à la F3. Ce qui fait la particularité de l'expérimentation I.N.R.A., c'est, d'une part l'utilisation de la Romanov comme race parentale $\left({ }^{*}\right)$, d'autre part la réalisation d'un programme rigoureux avec accouplements entre animaux de même génération sans sélection et mise en œuvre d'un nombre relativement élevé de béliers.

\section{Système de reproduction}

Le rythme original que nous avons adopté, permet d'apprécier la fertilité des antenaises à contre-saison et dans des conditions post-partum en saison. La fertilité observée à la $1^{\text {re }}$ lutte d'avril-mai à 15 mois $(0,86)$ étant identique à celle enregistrée sur les agnelles F1 en septembre-octobre à 8-9 mois (0,84 : RiCORDEAU et al., $1976 \mathrm{c})$, on pourrait admettre que la $1^{\text {re }}$ saison sexuelle des agnelles s'étale sur au moins 240 jours (septembre à avril), ce qui est une durée nettement supérieure à celles

(*) Cette race se caractérise, en plus de sa prolificité, par une faible mortalité embryonnaire (RICORDEAU et al., 1976 b, 1982) et une bonne viabilité en bergerie et en plein air (RICORDEAU et al., 1977 a ; Flamant et al., 1977). Dans les comparaisons eftectuées en France, la mortalité des agneaux Romanov est inférieure à celle des Finnois (RICORDEAU et al., 1977 b; DuboIs, 1979). Enfin, quel que soit le rythme ou le mode de reproduction, les brebis croisées, plus productives, s'adaptent très bien et ne «s'usent» pas plus rapidement que les brebis parentales rustiques (MARZIN et al., 1979 ; TCHAMITCHIAN et al., 1979). 

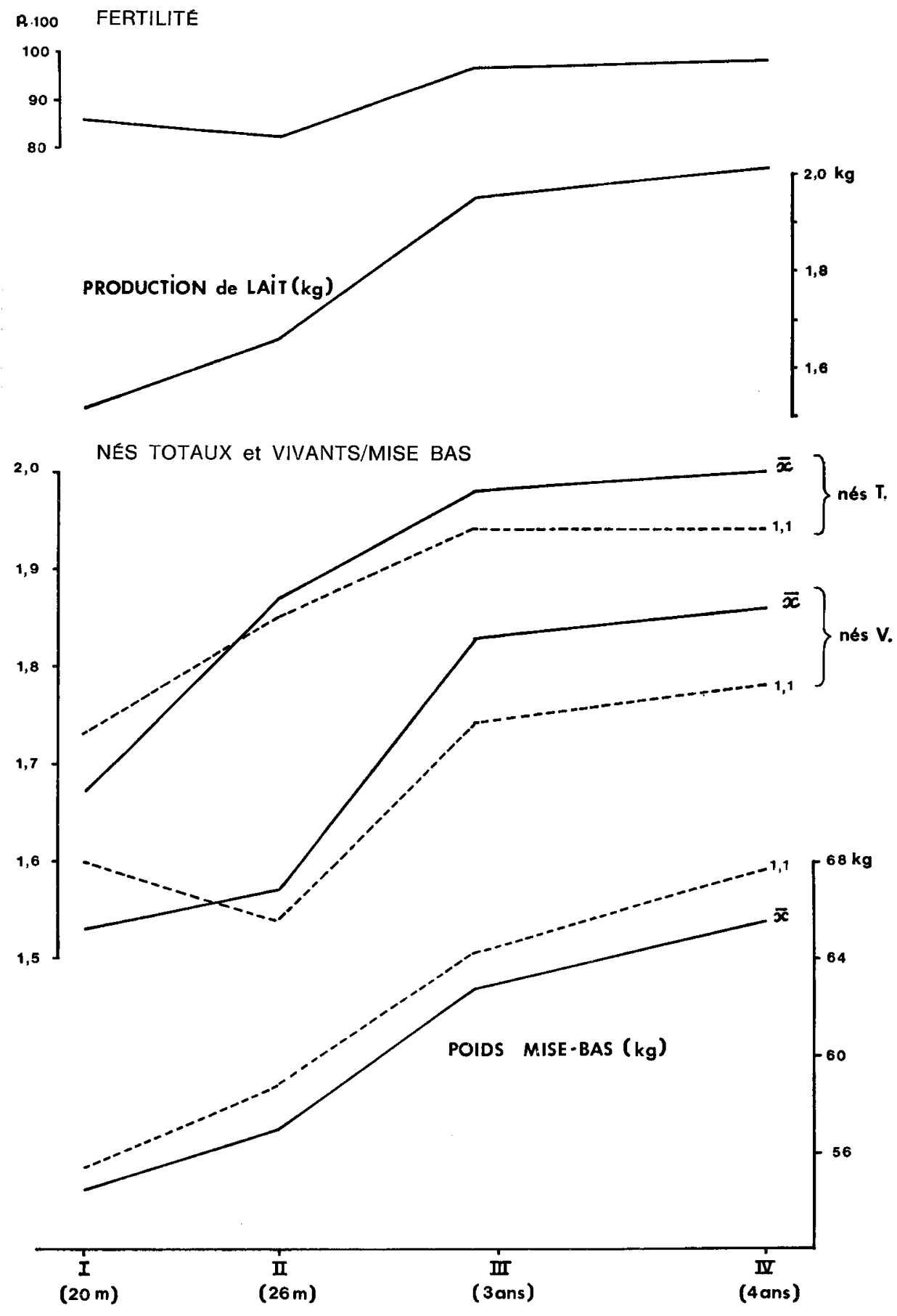

FIG. 3

$N^{*}$ de mise-bas(âge)

Performances des brebis $F 1$ à $F 4$, de la $1^{\text {re }}$ à la $4^{\circ}$ mise bas

$(1,1=$ agnelles nées et allaitées simples $; \bar{x}=$ moyenne générale $)$.

Performances of $F 1$ to $F 4$ ewes, from 1 st to 4 th lambing

$(1.1=$ ewe-lambs born single $; \vec{x}=$ total mean $)$. 
mesurées sur agnelles Romanov ou Finnoise en contrôle permanent d'œstrus (*). Puisque ces agnelles sont isolées des mâles depuis leur sevrage, il faut donc supposer que leur activité sexuelle a diminué bien avant le mois d'avril, mais que l'introduction des béliers a entraîné une augmentation importante et rapide de l'activité ovarienne et sexuelle, comme cela a bien été observé sur brebis Mérinos d'Australie ou Préalpes $d u S u d$, à peu près à la même période (OldhaM \& CoGNIE, 1980). Cependant, la distribution des mises bas ne révèle, de façon répétable, aucun pic de fécondation 15 à 25 jours après l'introduction des vasectomisés, aussi, en l'absence de contrôles d'œstrus, peut-on penser que «l'effet bélier » est peu marqué ou que le nombre de béliers disponibles pour les saillies est insuffisant pour féconder toutes les brebis venant en ostrus au début de la période de lutte. Il nous faut donc préciser l'activité sexuelle des antenaises à cette époque, afin d'obtenir une meilleure synchronisation des fécondations dès le début des saillies. Cela devrait également permettre d'améliorer la fertilité à la $2^{\text {e }}$ lutte post-partum de saison $(0,82)$, grâce à un allongement de la durée effective de lutte toujours trop courte pour les brebis agnelant tardivement ou allaitant deux agneaux.

\section{Effets maternels}

Cette étude met en évidence un effet maternel sur tous les poids jusqu'à 4 ans et sur la prolificité à la $1^{\text {re }}$ mise bas, comme si ce type de brebis privilégiait son niveau de productivité numérique ou ses qualités maternelles aux dépens de ses besoins de croissance pondérale (fig. 3 ).

Puisque les brebis nées et allaitées simples ont une taille de portée supérieure à celles nées doubles ou triples, il faudra tenir compte de ce facteur dans les programmes d'indexation des brebis. Notons que cet effet maternel sur la taille de portée a été observé sur brebis Mérinos d'Australie (PIPER \& MCGuirk, 1976 ; TURner, 1978), mais pas sur les brebis de la souche A.B.R.O. (MARTIN et al., 1981).

La production laitière pendant le premier mois qui suit la mise bas atteint $2 \mathrm{~kg}$ /jour à 3 ans. En fait, il s'agit là d'une valeur moyenne qui n'a pas grande signification, puisque les brebis se classent en 2 groupes bien distincts suivant qu'elles allaitent 1 ou 2 agneaux. Par exemple, à 3 ans (fig. 4), la production des brebis avec 2 agneaux varie de 1,5 à $3,0 \mathrm{~kg}$ autour d'une moyenne de $2,3 \mathrm{~kg}$, ce qui constitue une aptitude laitière importante qui pourrait encore être améliorée par sélection, si nécessaire.

\section{Paramètres génétiques}

L'équilibre de linkage n'étant pas atteint en F2 et F3, nos estimations n'ont qu'une valeur indicative, mais elles peuvent servir de référence avant l'application des programmes de sélection.

En ce qui concerne l'héritabilité, nos estimations sont assez conformes aux valeurs attendues. Par rapport à celles obtenues sur les brebis de la souche ABRO, elles sont très comparables pour les caractères de croissance (MARTIN et al., 1980), mais supérieures pour la prolificité (MARTIN et al., 1981).

(*) Durée de la saison sexuelle. Agnelles Romanov : 174 jours (Land et al., 1973); 157 et 185 jours (Thimonier, 1975). Agnelles Finnoises : 160 jours (Thimonier, 1975). Finnoises adultes : 190 et 230 jours (WHEEler et LAND, 1977); 228 jours (OsterberG, 1981). 


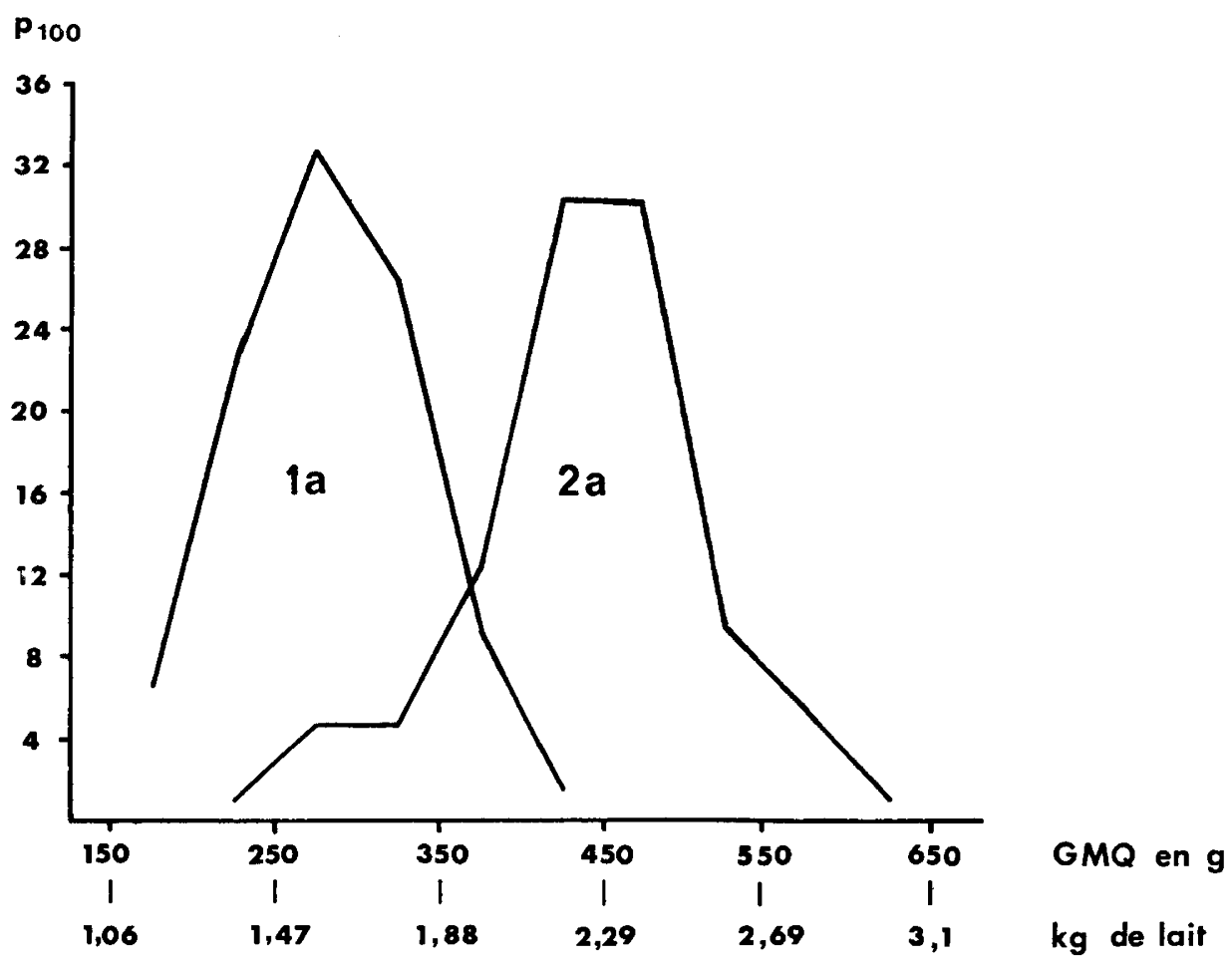

FIG. 4

Production laitière des brebis F2, F3 et F4 nées en 1977, à la $3^{\circ}$ mise bas à 3 ans (premier mois d'allaitement).

Brebis allaitant 1 agneau (1a) ou 2 agneaux (2a).

Milk production of F2, F3 and F4 ewes born in 1977 , at the 3rd lambing (3 years old), during the first month of lactation.

$1 a=$ ewes suckling one lamb

$2 a=$ ewes suckling two lambs.

La corrélation phénotypique entre le poids des agnelles à 70 et 90 jours et la prolificité à la mise bas $1(+0,11$ à 0,17$)$ est du même ordre que celle observée par Ch'ANG \& RAE (1972) entre poids au sevrage et prolificité $(0,13$ à 0,15$)$. Le poids à 90 jours et, plus particulièrement le gain 30-70 jours, sont en corrélation génétique positive avec la prolificité, alors que MARTIN et al. (1981) n'observent de relations positives qu'avec le poids des brebis avant la lutte ou après la mise bas. Une bonne vitesse de croissance semble également intéressante pour améliorer la durée de la première saison sexuelle, bien que les corrélations génétiques obtenues en NouvelleZélande sur la race Romney soient nettement positives pour CH'ANG \& RAE (1972) et légèrement négatives pour BAKER et al. (1979). 


\section{TABLEAU 11}

Création de souches synthétiques ou de nouvelles races croisées.

Synthetic lines or new breeds.

\begin{tabular}{|c|c|c|}
\hline Grande-Bretagne . & $\begin{array}{l}\text { A.B.R.O. Dam Line } \\
\text { (47\% Finnoise, } 24 \% \text { East Friesian, } \\
17 \% \text { Border L., } 12 \% \text { Dorset Horn }) \\
\text { Cambridge hybrid } \\
(25 \% \text { Finnoise, } 60 \% \text { Clun Forest, } \\
15 \% \text { pool d'autres races dont } \\
\text { Lleyn, Border L., Suffolk, ...) } \\
\text { British Milk and prolific sheep } \\
(70 \% \text { East Friesian, } 10 \% \text { Blueface } \\
\text { Leicester, } 10 \% \text { Poll Dorset, } 5 \% \\
\text { Lleyn, } 5 \% \text { Prolific breed) }\end{array}$ & $\begin{array}{l}\text { WIENER, } 1974 \text {; SMITH, } 1976 \text {; } \\
\text { SMITH et al., 1979; MARTIN et al., } \\
1980 \text { et } 1981 \text {; BoAZ et al., } 1981 . \\
\text { OWEN, } 1976 \text {; WILLIAMS et OWEN, } \\
1979 . \\
\text { DAVIES, } 1981 . \\
\text { ALDERSON, } 1980 .\end{array}$ \\
\hline Irlande $\ldots \ldots \ldots$ & $\begin{array}{l}\text { Fingalway (50\% Finnois, } 50 \% \text { Gal- } \\
\text { way) } \\
\text { Improved Galway strain I ( } 25 \% \\
\text { Finnois, } 75 \% \text { Galway) } \\
\text { New. I.G. Strain II (moins de } \\
25 \% \text { Finnois, Galway + Lleyn) }\end{array}$ & $\begin{array}{l}\text { HANRAHAN, } 1974 . \\
\text { FLANAGAN et al, } 1978 .\end{array}$ \\
\hline Espagne & $\begin{array}{l}\text { Souche Romanov } \times \text { Segureña } \\
\text { Scuche Romanov } \times \text { Aragonesa }\end{array}$ & $\begin{array}{l}\text { MARINE, } 1980 \\
\text { SYERRA, } 1980\end{array}$ \\
\hline Australie & $\begin{array}{l}\text { Gromark }(50 \% \text { Border L., } 50 \% \\
\text { Corriedale })\end{array}$ & GODLEE, 1976. \\
\hline $\begin{array}{l}\text { Nouvelle } \\
\text { Zélande ... }\end{array}$ & $\begin{array}{l}\text { Coopworth ( } 50 \% \text { Border L., } 50 \% \\
\text { Romney) } \\
\text { Souche «Whatawhata Hill C. " } \\
(50 \% \text { Border L., } 50 \% \text { Romney) } \\
\text { Croisées Booroola (croist. d'absorp- } \\
\text { tion des mâles Booroola sur des fe- } \\
\text { melles Romney) }\end{array}$ & $\begin{array}{l}\text { Coop, 1974, } 1978 . \\
\text { ClaRKE, 1962; Hight et al., } 1973 . \\
\text { KelLY et al., } 1980 .\end{array}$ \\
\hline U.S.A. & Dorset $\times$ Finnois & RINGWALl et al., 1980. \\
\hline
\end{tabular}

\section{Conclusion}

Finalement, cette expérimentation montre que la création d'une lignée synthétique est possible à deux conditions : utiliser le maximum de variabilité génétique et ne pas en perdre par une sélection prématurée avant obtention d'un maximum de recombinaisons, ou en éliminant des phénotypes indésirables mais plus productifs comme c'est le cas pour les animaux colorés (RicordeAu et al., 1982). La création de 
cette souche répond à une préoccupation nationale, mais aussi internationale, puisque des programmes de croisement ont été entrepris dans plusieurs pays ayant une population ovine importante (tabl. 11). Ces initiatives ont d'ailleurs des objectifs variés, puisqu'il s'agit soit de fabriquer des «mâles prolifiques » destinés à remplacer les béliers Border Leicester dans les schémas de croisement à double étage (cas des souches synthétiques créées en Grande-Bretagne), soit d'absorber des populations locales pour créer une nouvelle race de brebis (cas des souches sélectionnées en Australie ou Nouvelle-Zélande).

Dans le passé, les éleveurs français ont souvent eu recours au croisement pour créer de nouvelles races. Il s'agit là d'un outil dont l'utilisation doit être raisonnée, parallèlement aux efforts de sélection, en vue d'une efficacité maximum des plans d'amélioration génétique.

Reçu pour publication en février 1982.

\section{Remerciements}

Nous remercions R. Rouvier qui a lu cet article et suggéré d'utiles corrections.

\section{Summary}

Phenotypic and genetic parameters for growth and reproductive performance in crossbred ewes F1 to F4, born from the parental breeds Berrichon du Cher and Romanov

This study intends to compare the performance of growth and the maternal aptitudes of F1 to F4 ewes issue of crossbreeding between the two parental breeds Romanov and Berrichon du Cher. The least-squares analysis of variance has been achieved on 1298 ewes born from 1973 to 1977 and recorded until 1979. The original system of reproduction includes the first mating in may at 15 months (fertility : 0.86 ), the second post-partum mating in october at 20 months (fert. 0.82), and the following matings once a year in julyaugust (fert. 0.97 to 0.99 ). The recorded performances at lambings 1 to 4 are respectively : $1.67,1.87,1.98$ and 2.01 for the litter size; $54.5,57.0,62.8$ and $65.6 \mathrm{~kg}$ for the weight at lambing; $1.53,1.66,1.95$ and $2.01 \mathrm{~kg}$ for the average daily weight of milk suckled by the lambs during the first month of lactation.

The differences between generations F1 to F4 are significant for the weight at 70 and 90 days (given that the initial growth is lower in F1 ewe lambs) but not significant for the 30-70 day weight gain. They are not significant for the weight at lambing, the fertility, the number of lambs born and alive at lambing and the milk production of the ewes. This result confirms the previous observations obtained on F1 and F2 ewes, born from 1970 to 1972 and mated at the traditional time ; it demonstrates the lack of « regression 》 of performances from F1 to F4 in our conditions (Romanov as parental breed, no selection before the 4th generation).

The birth-rearing class of the ewe has a significant influence on the lambing weight until 4 years and on the litter size at first lambing, giving an advantage of 0.1 lamb in favour of single born ewes.

The heritability of performance has been estimated separately on F2 and F3 ewes by half sister anaiysis. The heritability of $30-70$ day weight gain $(0.17$ and 0.43$)$ is higher than the one relating the litter size $(0.09$ and 0.24$)$ and milk production $(0$ and 0.16$)$ at first lambing. The 30-70 day weight gain of ewe lambs is the character which has the higher genetic correlation with litter size at first lambing. 


\section{Références bibliographiques}

Alderson L., 1980. British Milksheep. The high performance breed. Prolific producer of lambs and milk. Farmers Weekly, février, 91-96.

Baker R.L., Clarke J.N., Carter A.H., Diprose G.D., 1979. Genetic and phenotypic parameters in New-Zealand Romney sheep. I - Body weights, fleece weights and oestrous activity. N.Z. J. agric. Res., 22, 9-21.

Bonz T.G., Jones R., Smith C., 1980. A note on a comparison of crossbreds from two prolific sheep breeds. Anim. Prod., 31, 323-325.

Ch'ANG T.S., RAE A.L., 1972. The genetic basis of growth, reproduction, and maternal environment in Romney ewes. II - Genetic covariation between hogget characters, fertility, and maternal environment of the ewe. Aust. J. agric. Res., 23, 149-165.

Clarke E.A., 1962. Crossbreeding in sheep. N.Z. J. agric., 105, 393-399.

Coop I.E., 1974. Crossbreeding, interbreeding and establishing a new breed of sheep. Proc. N.Z. Soc. anim. Prod., 34, 11-13.

Coop I.E., 1978. The Coopworth breed of sheep. World review of Anim. Prod., 14 (3), 9-15.

Davies R., 1981. Cambridge hybrid fulfils 20-year ambition. Farmers Weekly, mars 81.

Dickerson G., 1969. Experimental approaches in. utilising breed resources. Anim. breed. Abstr., 37, 191-202.

Dubors O., 1979. Expérimentation sur les races prolifiques Romanov et Finnoises dans les

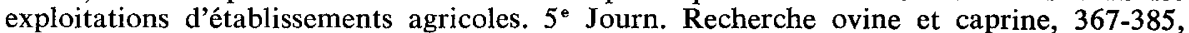
I.N.R.A.-I.T.O.V.I.C., Ed.

Flamant J.C., Jacquin M., Maquere M., 1977. Quelques aspects génétiques de la vitalité chez les agneaux. $3^{\mathrm{e}}$ Journ. Recherche ovine et caprine, 200-211 I.N.R.A.-I.T.O.V.I.C. Ed.

Flanagan S.P., Hanrahan J.P., O’Malley P., 1978. Prolific ewes increase cash income. Farm and Food Research, 10, 88-90.

GodleE A.C., 1976. Development of the Gromark. A new high performance dual purpose sheep breed. In Sheep Breeding Proc. Intern. Congr. Muresk and Perth., 189-194, Butterworths Ed. ( $2^{\mathrm{e}}$ éd.).

HanRahan J.P., 1974. Crossbreeding studies involving Finnish Landrace and Galway sheep. Proc. Working Symp. Zeist., 431-444.

HIGHT G.K., LAND D.R., JURY K.E., 1973. Hill country sheep production. V - Occurrence of oestrus and ovulation rate of Romney and Border Leicester $\times$ Romney ewe hoggets. N.Z. J. agric. Res., 16, 509-517.

Hulet C.V., Knight A.D., Ercanbrack S.K., 1981. The future for new or synthetic breeds or types of sheep. Int. Goat and Sheep Res., 1, 248-257.

Kelly R.W., Davis G.H., Allison A.J., 1980. Productive changes in Longwool breeds in New Zealand following crossbreeding with Booroola - type rams. Proc. Austr. Soc. anim. Prod., 13, 413-416.

Land R.B., Pelletier J., Thimonier J., Mauleon P., 1973. A quantitative study of genetic difference in the incidence of oestrus, ovulation and plasma luteinizing hormone concentration in the sheep. J. Endocr., 58, 305-317.

Marine A., 1980. Communication personnelle.

Martin T.G., Sales D.I., Smith C., Nicholson D., 1980. Phenotypic and genetic parameters for lamb weights in a synthetic line of sheep. Anim. Prod., 29, 261-269. 
Martin T.G., Nicholson D., Smith C., Sales D.I., 1981. Phenotypic and genetic parameters for reproductive performance in a synthetic line of sheep. J. agric. Sci., 96, 107-113.

Marzin J., Prud'hon M., Brelurut A., Angevain J., Reboul G., 1979. Performances des brebis de races locales ou croisées Romanov soumises à un rythme de reproduction accéléré. $5^{\mathrm{e}}$ Journ. Recherche ovine et caprine, 349-366. I.N.R.A.-I.T.O.V.I.C., Ed.

Oldham C.M., Cognie Y., 1980. Do ewes continue to cycle after teasing ? Proc. Aust. Soc. anim. Prod., 13, 82-85.

Oltenacu E.A.B., Boylan W.J., 1981 a. Productivity of purebred and crossbred Finnsheep. I. - Reproductive traits of ewes and lamb survival. J. anim. Sci., 52, 989-997.

Oltenacu E.A.B., Boylan W.J., 1981 b. Productivity of purebred and crossbred Finnsheep. II. - Lamb weights and production indices of ewes. J. anim. Sci., 52, 998-1006.

Osterberg S., 1981. Breeding season of the Finnsheep ewe. Acta Agric. Scand., 31, 11-16.

OWEN J.B., 1976. The development of a prolific breed of sheep. 27th Annual Meeting E.A.A.P., Zurich, 23-26 août.

Pattie W.A., Smith M.D., 1964. A comparison of the production of F1 and F2 Border Leicester $\times$ Merino ewes. Austr. J. exp. agric. anim. Husb., 4, 80-85.

PIPER L.R., McGuiRK B.J., 1976. Age effects on the difference in reproductive performance between twin and single born Merino ewes. Aust. Soc. anim. Prod., 105-108.

Ricordeau G., Boccard R., 1961. Relations entre la quantité de lait consommé par les agneaux et leur croissance. Ann. Zootech., 10, 113-125.

Ricordeau G., Tchamitchian L., Eychenne F., Razungles J., 1976 a. Performances de reproduction des brebis Berrichonnes du Cher, Romanov et croisées. I. - Activité sexuelle en début de saison et à contre saison. Ann. Génét. Sél. anim., 8, 9-24.

Ricordeau G., Razungles J., Eychenne F., Tchamitchian L., 1976 b. Peiformances de reproduction des brebis Berrichonnes du Cher, Romanov et croisées. II. - Composantes de la prolificité. Ann. Génét. Sél. anim., 8, 25-35.

Ricordeau G., Desvignes A., Tchamitchian L., Rastogi R., Lefevre C., 1976 c. Amélioration de la productivité des brebis Berrichonnes du Cher (BC) par croisement. I. - Productivité numérique des brebis BC, Cotentin, Border Leicester, Romanov et des trois types de F1. Ann. Génét. Sél. anim., 8, 367-389.

Ricordeau G., Tchamitchian L., Lefevre C., Brunel J.C., Desvignes A., $1976 \mathrm{~d}$. Amélioration de la productivité des brebis Berrichonnes du Cher (BC) par croisement. III. - Performances de reproduction des trois premières générations de croisées entre les races BC et Romanov. Ann. Génét. Sél. anim., 8, 405-419.

Ricordeau G., Tchamitchian L., Lefevre C., Brunel J.C., 1977 a. Amélioration de la productivité des brebis Berrichonnes du Cher par croisement. IV. - Durée de gestation et viabilité des agneaux Berrichons, Romanov et croisés F1, F2 et F3. Ann. Génét. Sél. anim., 9, 219-239.

Ricordeau G., Tchamitchian L., Lefevre C., Brunel J.C., 1977 b. Différences génétiques de viabilité entre agneaux de bergerie Romanov, Berrichon du Cher, F1 Romanov $\times$ Berrichon et Finnois. $3^{\circ}$ Journ. Recherche ovine et caprine, 189-199. I.N.R.A.-I.T.O.V.I.C. Ed.

Ricordeau G., Razungles J., Lajous D., 1982. Heritability of ovulation rate and level of embryonic losses in Romanov breed. 2nd World. Cong. Genet. Liv. Prod. Madrid. VII, 591-595.

Ringwall K.A., Whiteman J.V., Fields J.E., Spencer R.J., 1980. Developing a fertile line of fall lambing Dorset $\times$ Finnish Landrace sheep : preliminary observations. Ann. Sci. Report. Oklahoma, 19-20.

SierRa A.I., 1980. Formacion de una nueva raza ovina sintetica a partir de las razas Romanov y raza Aragonesa. Zootechnia., XXXI, 14-20. 
Sмiтн C., 1976. The Dam line. Sires for mothers of meat lamb. A.B.R.O. Report, p. 45 (short notes).

Smith C., King J.W.B., Nicholson D., Wolf B.T., Bampton P.R., 1979. Performance of crossbred sheep from a synthetic Dam line. Anim. Prod., 29, 1-9.

TChamitchian L., 1975. Stratégie d'utilisation des races prolifiques en race pure et en croisement. $1^{\text {res }}$ Journ. Recherche ovine et caprine, (II), 82-102. I.N.R.A.-I.T.O.V.I.C., Ed.

Tchamitchian L., Ricordeau G., Lefevre C., Brunel J.C., Lajous D., 1979. Amélioration de la productivité numérique par croisement. $5^{\ominus}$ Journ. Recherche ovine et caprine, 323-348. I.N.R.A.-I.T.O.V.I.C., Ed.

Thimonier J., 1975. Etude de la puberté et de la saison sexuelle chez les races prolifiques et leurs croisements avec les races françaises. $1^{\text {res }}$ Journ. Recherche ovine et caprine (II), 18-37. I.N.R.A.-I.T.O.V.I.C., Ed.

TURNER H.N., 1978. Selection for reproduction rate in Australian sheep; direct responses. Austr. J. agric. Res., 29, 327-350.

WheEler A.G., LAND R.B., 1977. Seasonal variation in oestrus and ovarian activity of Finnish Landrace, Tasmanian Merino and Scottish Blackface ewes. Anim. Prod., 24, 363-376.

Wiener G., 1974. Crossbreeding studies with sheep at the A.B.R.O. Edimburgh. Proc. Working Symp. Zeist, 493-510.

WILliams G.L., OWEN J.B., 1979. Co-operative group breeding schemes for prolific lowland sheep breeds. 30th Annual Meet. E.A.A.P., Harrogate. 